\section{Medical \\ Bacteriology: a Practical Approach}

\author{
Peter Hawkey \\ and Diedre Lewis, editors
}

\section{Oxford University Press \\ Oxford, United Kingdom \\ ISBN: 0-19-963778-4}

Pages: 409, Price: US\$59.50

Medical Bacteriology is a multicontributor work with chapters provided by various expert medical microbiologists from the United Kingdom. The information is organized into 4 basic areas totaling 12 chapters; 6 covering the analysis of various types of patient specimens; 2 chapters on antimicrobial analysis, susceptibility testing, and direct assay in patient specimens; 2 chapters on laboratory management issues, including information technology, quality control, and quality assurance; and 2 chapters on the role of the laboratory in hospital infection control programs and in the support of epidemiologic investigations. Distributed throughout the text are 81 individual testing protocols, which can be found either by referring to a table at the front of the book or by searching the index. Also included are 4 appendixes.

Multiple options exist for presenting the fundamentals of medical bacteriology. Some texts have used a disease-based approach. Others have used an organism-based approach. Medical Bacteriology takes a specimen-based approach: bacterial diseases and their causative agents are addressed through the proper collection, processing, and analysis of clinical specimens. From a laboratory perspective, the specimen-based approach has substantial advantages. In this text, the critical importance of proper collection and transport of specimens is clearly communicated for each specimen type, and appropriate protocols are provided. In addition, an in-depth discussion is presented on the proper interpretation of cultures and other laboratory findings for each specimen type. For example, the chapter on urine bacteriology contains not only guidelines for interpreting various types of urine cultures, but also explains the relevance of other routine urinalysis findings to infections of the urinary tract. One disadvantage of the specimen-based approach is that organism identification protocols may appear in multiple chapters.

The chapters devoted to antimicrobial issues provide basic testing protocols and valuable insights on the selection of appropriate agents for routine testing and reporting. The relative advantages and disadvantages of diffusion versus dilution methods are clearly described, and readers from the United States may find some of the alternatives to the standard KirbyBauer procedure to be of interest.

The chapters devoted to laboratory management provide excellent insights on the rapidly evolving field of laboratory information technology. Laboratorians should find the comparison of stand-alone "legacy" systems with more integrated hospitalwide and health system-wide designs useful in making decisions on laboratory information management.

The chapters on infection control and epidemiology provide an overview of the technical and administrative issues encountered by the laboratory. The organization and function of hospital infection control committees are discussed, and guidance for performing basic cohort and case-control studies is presented.

Emphasis is placed on the processing and analysis of specimens with well-established protocols and materials. Bacterial identification and characterization protocols are based on techniques that have been in general use for many years. Readers interested in newer approaches and in analyses of some of the more exotic bacterial zoonoses are provided with appropriate references.

The book flows smoothly from chapter to chapter. Similar material appearing in various chapters is appropriately cross-referenced. The text is clearly written, with jargon and acronyms kept to a minimum. At 409 pages, including the appendixes and index, this book can be easily read in a few sittings, and readers, including students, technologists, laboratory supervisors, and senior scientists, should find it to be a useful reference.

\section{Robbin S. Weyant*}

*Centers for Disease Control and Prevention, Atlanta, Georgia, USA

Address for correspondence: Robbin S. Weyant, Centers for Disease Control and Prevention, 1600 Clifton Rd, Mailstop A17, Atlanta, GA 30333, USA; fax: 404-639-0883; email: rweyant@cdc.gov

\section{Cases in Human Parasitology}

\section{Judith S. Heelan}

\section{ASM Press, Washington, DC ISBN: 1-55581-296-1}

\section{Pages: 243, Price: US\$59.95}

This compact, glossy, paperbound text contains 62 cases in 5 sections: I) Intestinal Protozoa, II) Blood and Tissue Protozoa, III) Cestodes, Trematodes and Intestinal Nematodes, IV) Blood and Tissue Nematodes, and V) Challenging Cases. The intent was to emphasize the relationship between diagnosis and patient care. This goal is laudable; unfortunately, serious shortcomings limit the 
book's usefulness for students, professors, or laboratorians.

A major challenge is the format: the presented case is "textbook," and the "answer" is given in the case presentation, leaving little need for the answer section. How many junior parasitologists or biologists with casual interest in parasites don't know that bile-stained, barrel-shaped nematode eggs with prominent polar plugs represent Trichuris eggs? If the clinical history and illustrations are presented and the detailed description of the organism is left to the answer, the reader can look at the illustrations, decipher the morphologic features, consider the possibilities, and then differentiate by using existing features.

Some case presentations had no illustrations, which is a prerequisite. Most illustrations were adequate, but some were unacceptable. Figure 4.1 presumes to illustrate an Entamoeba histolytica cyst, but the diagnosis could not be made from the image. Figure 16.1 is listed as typical of Babesia infection, yet after close study, if Babesia organisms are present, they are not typical. The illustrations of microsporidia at low-power magnification were perplexing. Use of identical images to illustrate East African and West African trypanosomes is unacceptable. The illustration for case 52 (onchocerciasis) shows a Giemsa-stained microfilaria with a sheath. The morphologic features and the sheath stained with Giemsa indicate a Brugia microfilaria, not an Onchocerca microfilaria.

In case 52 (Onchocerca), surgical removal of regional lymph nodes is advised, in addition to removal of nodules containing adult worms. This is not standard medical advice. In case 48 (dracunculiasis), it is stated that cisterns in Iran and step wells in India are common sources of infection and that prevalence of this infection has been reduced in most areas, except India, Pakistan, and a few countries in Africa. Guinea worm has been absent from Iran since 1972, from Pakistan since 1993, and from India since 1996. In the same case study, it is stated that metronidazole is often used to complement or replace traditional removal of worms, and that niridazole, thiabendazole, and mebendazole are also useful. None of these drugs has any benefit in Guinea worm infection treatment. In case 3 (cyclosporiasis), it is stated that infections from ingestion of contaminated fruits, such as imported strawberries, have been reported. Not true; strawberries have never been implicated.

Given the multiple errors and lack of attention to detail (Colombia is misspelled; the width of Anisakis L3 is given as $1 \mathrm{~cm}$ ), this book has little to offer, despite its reasonable price (\$60). This is unfortunate because a well-done series of teaching cases could fill a much needed void.

\section{Mark L. Eberhard*}

*Centers for Disease Control and Prevention, Atlanta, Georgia, USA

Address for correspondence: Mark L. Eberhard, Centers for Disease Control and Prevention, 1600 Clifton Rd, Mailstop F22, Atlanta, GA, 30333, USA; fax: 770-488-7794; email: mle1@cdc.gov

\section{Correction: Vol. 11, No. 4}

In "Methicillin-resistant Staphylococcus aureus Toxic Shock Syndrome" by Sophie Jamart et al., an error occurred. In a listing of laboratory results (third paragraph, fifth sentence), cyclic AMP receptor protein $43.7 \mathrm{ng} / \mathrm{mL}$ should be C-reactive protein $43.7 \mathrm{mg} / \mathrm{dL}$.

The corrected article appears online at http://www. cdc.gov/ncidod/EID/vol11no04/04-0893.htm

We regret any confusion this error may have caused.

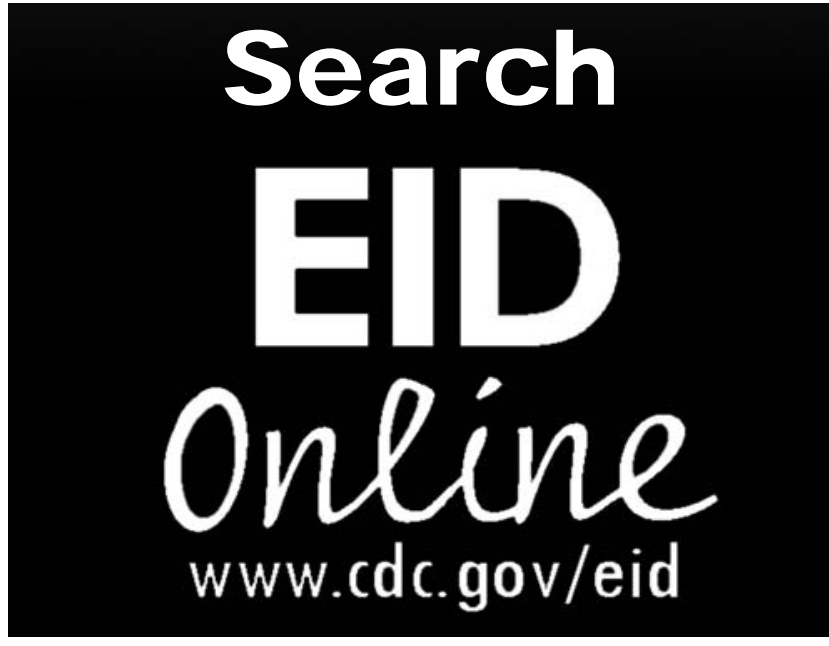

